\title{
Reconstruction-based multivariate contribution analysis for fault isolation: A branch and bound approach
}

\author{
Bo He ${ }^{\mathrm{a}}$, Xianhui Yang ${ }^{\mathrm{a}}$, Tao Chen ${ }^{\mathrm{b},{ }^{*}}$, Jie Zhang ${ }^{\mathrm{c}}$ \\ ${ }^{a}$ Department of Automation, Tsinghua University, Beijing 100084, China \\ ${ }^{\mathrm{b}}$ Division of Civil, Chemical and Environmental Engineering, University of Surrey, Guildford GU2 7XH, UK \\ ${ }^{\mathrm{c}}$ School of Chemical Engineering and Advanced Materials, Newcastle University, Newcastle upon Tyne NE1 7RU, \\ UK \\ * Corresponding author; Tel.: +44 1483 686593; Email: t.chen@ surrey.ac.uk
}

\begin{abstract}
Identification of faulty variables is an important component of multivariate statistical process monitoring (MSPM); it provides crucial information for further analysis of the root cause of the detected fault. The main challenge is the large number of combinations of process variables under consideration, usually resulting in a combinatorial optimization problem. This paper develops a generic reconstruction based multivariate contribution analysis (RBMCA) framework to identify the variables that are the most responsible for the fault. A branch and bound $(\mathrm{BAB})$ algorithm is proposed to efficiently solve the combinatorial optimization problem. The formulation of the RBMCA does not depend on a specific model, which allows it to be applicable to any MSPM model. We demonstrate the application of the RBMCA to a specific model: the mixture of probabilistic principal component analysis (PPCA mixture) model. Finally, we illustrate the effectiveness and computational efficiency of the proposed methodology through a numerical example and the benchmark simulation of the Tennessee Eastman process.
\end{abstract}

Keywords- Branch and bound; Combinatorial optimization; Fault detection and isolation; Multivariate contribution analysis; Multivariate statistical process control

\section{Introduction}

Fault detection and diagnosis (FDD) have become more and more important to ensure safe, efficient and environmentally benign operation of complex industrial processes [1,2]. Meanwhile, with the wide use of distributed control system with automatic data acquisition, huge amount of process data are routinely collected, making data driven FDD methods such as multivariate statistical process monitoring (MSPM) possible [3]. Traditional MSPM approaches via principal component analysis (PCA) or partial least squares (PLS) have been used successfully in many applications [4,5,6]. The concept of these PCA/PLS-based MSPM methods is to project the original data onto a low dimension subspace which captures most of the process variation under normal operation conditions (NOC). Then, two monitoring statistics ( $\mathrm{T}^{2}$ and SPE) and their confidence bounds (control limits) are constructed under the assumption that the process data are Gaussian [7]. When data are not Gaussian distributed, notable solutions to MSPM include kernel density estimation [8], Gaussian mixture model (GMM) [9,10,11], independent component analysis [12], one-class SVM model [13], among others. The probabilistic PCA (PPCA) mixture model is an extension of GMM by incorporating a probabilistic version of PCA, and it has been shown to be effective for MSPM [14,15]. The probabilistic formulation used by the PPCA mixture model provides a unified likelihood-based statistic that offers clearer monitoring result. 
Fault isolation is the "downstream" step of fault detection in MSPM. The main task of fault isolation is to identify the most responsible variables for the detected fault. The isolated faulty variables may further serve as the basis for more in-depth root cause analysis and fault diagnosis [16]. The most popular method of fault isolation for traditional PCA model is contribution analysis [17]. The contributions of each process variable to the monitoring statistics are calculated. The variables with high contribution are considered to be the faulty variables. This basic contribution analysis has been improved in various studies to consider multiple process variables simultaneously [18]. Reconstruction based contribution was proposed specifically for PCA to re-calculate the variables and monitoring statistics along "faulty directions" [19,20,21]. The variables or directions are considered faulty if the reconstructed monitoring statistics are less than control limits, i.e. replacing these variables with the reconstructed values would bring the process back to normal. Similarly, a missing variable contribution analysis was proposed for probabilistic formulation of PCA and the PPCA mixture model [14]. In this method, each variable is treated as if it was missing and the expectation (with respect to the missing variable) of monitoring statistic is re-calculated. The variable corresponding to the largest reduction of monitoring statistic is considered to be the faulty variable. However, this approach is originally not suitable for studying the joint contribution of multiple variables due to the large number of possible variable combinations that are required to be evaluated. Later, a branch and bound (BAB) algorithm was proposed to efficiently solve this problem for the case of a single PPCA model [22].

$\mathrm{BAB}$ is a widely used method for solving combinatorial optimization problems. The key idea is to examine the bound of the objective function. For a maximization problem, if the upper bound on a subset of candidate solutions is less than the lower bound of the optimum, then this subset can be discarded. Compared with exhaustive search, $\mathrm{BAB}$ can dramatically save computation through the removal of subsets without actually evaluating their objective function. The primary task of BAB is thus the design of appropriate bounds and corresponding branching strategies, both of which depend on specific applications. In the context of fault isolation, existing studies imply that for different process models, different lower/upper bounds would have to be developed to enable the implementation of the $\mathrm{BAB}$ method. Considering the large number of process models proposed in the literature, the lack of a general solution has become the major obstacle to the application of $\mathrm{BAB}$ for fault isolation.

Building on these existing developments, this paper proposes a general framework for reconstruction based multivariate contribution analysis (RBMCA) and the corresponding solution strategy by using the BAB method. Specifically, the contribution of multiple process variables to a detected fault is evaluated by following the reconstruction and missing variable approach [14,19,20,21]. In order to find the combination of variables that are the most responsible to the fault, a combinatorial optimization problem is formulated, followed by the development of a feasible solution set and bounds to enable the application of BAB. The present study is similar to the previous work [22] in that both aim to use $\mathrm{BAB}$ for isolating multiple faulty variables. The major difference is that the previous work is focused on devising a specific $\mathrm{BAB}$ algorithm for a particular monitoring model (PPCA), whilst the current paper proposes a generic approach that can be used for any monitoring models, and thus significantly extends the applicability of multivariate contribution analysis. We demonstrate the application of the proposed RBMCA framework to the PPCA mixture model for process monitoring through an illustrative numerical example and the simulated Tennessee Eastman benchmark process.

The rest of this paper is organized as follows. The generic RBMCA framework for fault isolation is presented in section 2. In section 3, the application of RBMCA to PPCA mixture model is introduced. Section 4 presents the case studies and finally section 5 concludes this paper.

\section{The generic RBMCA framework}

2.1 Problem formulation

Suppose that a statistical model has been developed to represent the process data under NOC, and appropriate 
monitoring statistics with corresponding confidence bounds have been established. Without losing generality, we denote the monitoring statistic in terms of the likelihood of $n$-dimensional data $\mathbf{x}$ under the model $\mathbf{M}: \mathrm{p}(\mathbf{x} \mid \mathbf{M})$, and the confidence bound $h$. Clearly, when $\mathrm{p}(\mathbf{x} \mid \mathbf{M})>h$, the process data conforms to the NOC model whereas when $\mathrm{p}(\mathbf{x} \mid \mathbf{M})<h$, the process is classified as out-of-control. Notice that for classic monitoring statistics, like $\mathrm{T}^{2}$ or SPE, the above inequality relations must be reversed. Nevertheless, we adopt the likelihood-based monitoring statistic to facilitate the application of the RBMCA methodology to a probabilistic process model in section 3.

If the process data $\mathbf{x}$ is identified as faulty, i.e. $\mathrm{p}(\mathbf{x} \mid \mathbf{M})<h$, the task is to find out which variables are contributing the most to the fault. Following the concept of variable reconstruction $[19,20,21]$ (or similarly missing variable approach [14]), the contribution of a subset of $d$-dimensional variables $(d<n), \mathbf{x}_{m}$, can be evaluated through the reconstructed monitoring statistic. Let the faulty data be divided as $\mathbf{x}=\left[\mathbf{x}_{m}, \mathbf{x}_{o}\right]$ where $\mathbf{x}_{\mathrm{o}}$ is "observed"; the "missing" variables $\mathbf{x}_{m}$ can be reconstructed by maximizing the likelihood (i.e. the monitoring statistic) $\mathrm{p}\left(\mathbf{x}_{m}, \mathbf{x}_{o} \mid \mathbf{M}\right)$. Correspondingly, the reconstructed likelihood is

$$
\mathrm{p}\left(\mathbf{x}_{m}^{*}, \mathbf{x}_{o} \mid \mathbf{M}\right)_{\mathrm{rec}}=\max _{\mathbf{x}_{m} \in \mathbf{R}^{d}} \mathrm{p}\left(\mathbf{x}_{m}, \mathbf{x}_{o} \mid \mathbf{M}\right)
$$

The contribution of variables $\mathbf{x}_{\mathbf{m}}$ may be further defined as the increase of likelihood from reconstruction:

$$
\operatorname{RBMVC}_{\mathbf{x}_{m}}=\mathrm{p}\left(\mathbf{x}_{m}^{*}, \mathbf{x}_{o} \mid \mathbf{M}\right)_{\text {rec }}-\mathrm{p}(\mathbf{x} \mid \mathbf{M})
$$

In principle, the maximization problem in eq. (1) can be solved using numeric optimization methods. In many cases, the solution can be simplified by exploiting the special characteristics of the chosen model $\mathbf{M}$, and this will be demonstrated in Section 3.

The main challenge in RBMCA is thus not the reconstruction of $\mathbf{x}_{m}$ per se, but the search for the subset of variables that should be reconstructed. More rigorously, let $X_{n}=\left\{x_{1}, x_{2}, \cdots, x_{n}\right\}$ denote the ensemble of all $n$ variables. The objective of RBMCA is to find out the missing variable set $X_{m} \subset X_{n}$ such that $\phi\left(X_{m}\right)=\mathrm{p}\left(\mathbf{x}_{m}^{*}, \mathbf{x}_{o} \mid \mathbf{M}\right)_{\text {rec }}$ reaches maximum, i.e. these variables contribute the most significantly to the occurrence of the fault. However, this formulation has a trivial solution by simply reconstructing all process variables. Due to the maximization in eq. (1), adding more variables as missing will always be desired (formal proof is given as Proposition 1). Conceptually, the number of variables identified as faulty should be constrained to facilitate further fault diagnosis. This conceptual preference can be realized by constraining solutions within the following set of feasible solutions:

$$
S=\left\{X_{m} \mid \forall x_{i} \in X_{m}, \phi\left(X_{m} \backslash x_{i}\right) \leq h, \phi\left(X_{m}\right)>h, X_{m} \subset X_{n}\right\}
$$

The condition $\phi\left(X_{m}\right)>h$ expresses that the reconstructed likelihood should bring the process back to normal, while $\phi\left(X_{m} \backslash x_{i}\right)$ means that by excluding any single variable, the reconstructed likelihood indicates out-of-control. In essence, the set $S$ includes all solutions that make the process normal with as fewer variables being missing as possible. Therefore, the RBMCA is to find the optimal solution $X_{m}^{*}$ such that

$$
\phi\left(X_{m}^{*}\right)=\max _{X_{m} \in S} \phi\left(X_{m}\right)
$$

This is a combinatorial optimization problem with $2^{\text {n }}$ candidates, which grow exponentially with $n$ thus an exhaustive search is infeasible. Next, a BAB algorithm is presented to solve this problem efficiently.

\subsection{Solution using BAB method}

To solve the optimization problem in eq. (4), we propose an upward BAB algorithm as illustrated in the solution tree in Fig. 1 for a problem of five variables. Each node represents a candidate solution. The algorithm 
starts from the root node, corresponding to all variables being missing, and gradually makes some missing variables observed when it searches upward. The label of each node in the solution tree denotes the variable becoming observed when following the search path. For example, the sub-node of the root with label "1" (search path "root $\rightarrow 1$ ") refers to missing variables $\left\{x_{2}, x_{3}, x_{4}, x_{5}\right\}$, and its sub-node with label "2" (search path "root $\rightarrow 1 \rightarrow 2$ ") means missing variables $\left\{x_{3}, x_{4}, x_{5}\right\}$. Branches of each node are non-overlapping sub-problems (sub-nodes) of the original problem (parent node). Bound of the objective function is determined from nodes that have been examined. If any node has a smaller objective value (for maximization) than the lower bound, this sub-problem cannot be optimal and this branch can be pruned; otherwise continue the search from the current node to the sub-nodes (termed "branching" in $\mathrm{BAB}$ ). The bounding and pruning of branches help find the optimal solution more efficiently than an exhaustive search. Meanwhile, the BAB algorithm guarantees that the solution is a global optimum, in contrast to many heuristic search algorithms.

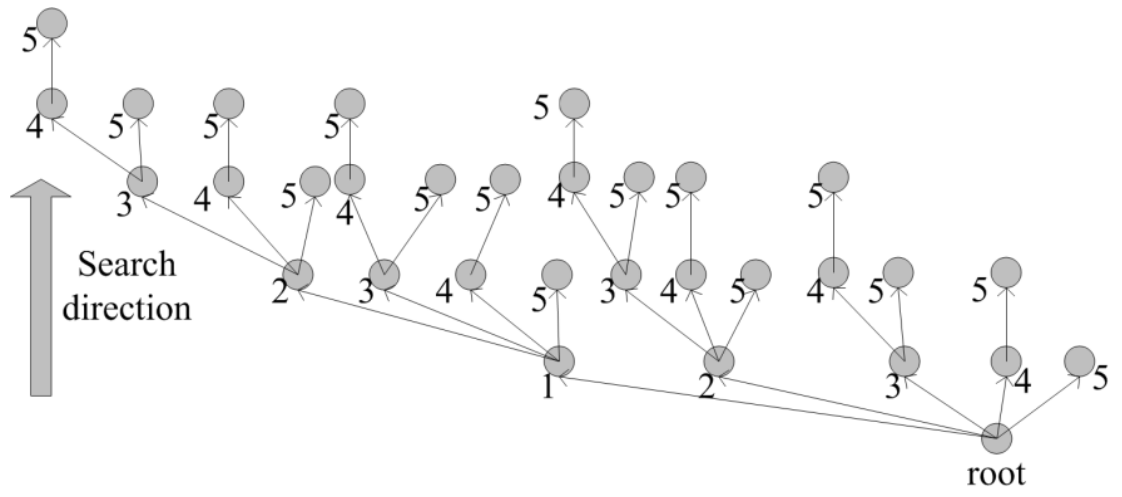

Fig. 1. Solution tree for five process variables.

The solution tree in Fig. 1 is asymmetric, that is, the missing variables at a particular node cannot all become observed in its sub-nodes during branching. For example, the node via path "root $\rightarrow 2$ " has missing variables $\left\{x_{1}, x_{3}, x_{4}, x_{5}\right\}$, but it cannot further branch to make $x_{1}$ observed. Otherwise, the path "root $\rightarrow 2 \rightarrow 1$ " would be a replicate of a previously branched path "root $\rightarrow 1 \rightarrow 2$ ". To handle this situation, at each node of the solution tree, we define $X_{\mathrm{c}}$ the candidate set consisting of all the missing variables that can become observed in sub-nodes:

$$
X_{c}=\left\{x_{j+1}, x_{j+2}, \ldots, x_{n}\right\} \subset X_{m}
$$

where $j$ is the largest index of variables that are observed at this node. Consequently for the root node, $X_{m}=X_{n}$, $X_{c}=X_{n}$ and $X_{o}=\varnothing$. For simplicity, we use the missing variables set $X_{m}$ to indicate the corresponding node hereafter. Furthermore, we define the set of all sub-nodes of node $X_{m}$ :

$$
\Gamma=\left\{X_{m} \backslash X_{f} \mid X_{f} \subset X_{c}\right\}
$$

To apply the BAB approach to this problem, the upper and lower bounds of the objective function over all elements of $\Gamma$ are required, and they both can be derived from Proposition 1 below.

\section{Proposition 1.}

Consider a node with the set of missing variables $X_{m}$ and candidate set $X_{c} . \forall x_{i} \in X_{c}, \quad \phi\left(X_{m} \backslash x_{i}\right) \leq \phi\left(X_{m}\right)$.

Proof. Let $\mathbf{x}_{m}=\left[\mathbf{x}_{m l i}, x_{i}\right]$ be the missing variable set. From the principle of optimization:

$$
\phi\left(X_{m}\right)=\max _{\mathbf{x}_{m}} p\left(\mathbf{x}_{m}, \mathbf{x}_{o} \mid \mathbf{M}\right)=\max _{\mathbf{x}_{m \backslash i}, x_{i}} p\left(\mathbf{x}_{m \backslash i}, x_{i}, \mathbf{x}_{o} \mid \mathbf{M}\right) \geq \max _{\mathbf{x}_{m \backslash i}} p\left(\mathbf{x}_{m \backslash i}, x_{i}, \mathbf{x}_{o} \mid \mathbf{M}\right)=\phi\left(X_{m} \backslash x_{i}\right)
$$

Proposition 1 suggests that the upper bound over all elements of $\Gamma$ is $\phi\left(X_{m}\right)$, and the lower bound is $\phi\left(X_{m} \backslash X_{c}\right)$. Therefore, non-optimal nodes can be pruned based on $\phi\left(X_{m}\right)$ and $\phi\left(X_{m} \backslash X_{c}\right)$. 
First, assume that $B$ is the lower bound of global optimum. If $\phi\left(X_{m}\right)<B$, this node and all its sub-nodes cannot be the optimum and thus can be pruned.

Furthermore, if $\phi\left(X_{m} \backslash X_{c}\right)>h$, none of its sub-nodes belongs to $S$ except for the upmost node of this branch, i.e. $X_{m} \backslash X_{c}$. In addition, if $\phi\left(X_{m} \backslash X_{c}\right)>h$, all unvisited sub-nodes belonging to the parent node of $X_{m}$ can be pruned (i.e., the brother nodes (and their sub-nodes) to the right of $X_{m}$ in the solution tree). This statement can be verified as follows. Let $j$ be the largest index of variables that are observed at the current node $\left(X_{m}\right)$. The structure of the solution tree in Fig. 1 shows that the parent node of $X_{m}$ is $X_{m} \cup x_{j}$. Thus, the $p$-th unvisited brother node of

$X_{m}$ is $X_{m}^{p}=\left\{X_{m} \cup x_{j}\right\} \backslash x_{j+p}$ and the corresponding candidate set is $X_{c}^{p}=\left\{x_{j+p+1}, \ldots x_{n}\right\} \subset X_{c}, p=\{1, \ldots, n-j-1\}$. The lower bound of $X_{m}^{p} \quad$ is $\phi\left(X_{m}^{p} \backslash X_{c}^{p}\right)=\phi\left(\left\{X_{m} \cup x_{j}\right\} \backslash\left\{x_{j+p}, x_{j+p+1}, \ldots x_{n}\right\}\right), p=\{1, \ldots, n-j\}$. It can be seen that $\left\{X_{m}^{p} \backslash X_{c}^{p}\right\} \supset\left\{X_{m} \backslash X_{c}\right\}$, and according to Proposition $1 \phi\left(X_{m}^{p} \backslash X_{c}^{p}\right) \geq \phi\left(X_{m} \backslash X_{c}\right)>h$. This suggests $\left\{X_{m}^{p} \backslash X_{c}^{p}\right\} \notin S$.

Consequently, none of the unvisited brother nodes and their sub-nodes belongs to the set of feasible solutions $S$.

Finally, additional efficiency can be gained by sorting the variables in descending order according to their univariate contribution to the occurrence of fault. A large univariate contribution indicates that the variable is heavily influenced by the fault and should be examined preferentially.

In summary, the complete RBMCA algorithm runs as follows.

Sort the variables in descending order according to their individual contribution to the fault. Initialize the root node with $X_{m}=X_{n}, X_{c}=X_{n}$ and $X_{o}=\varnothing$. Set the lower bound $B$ to an arbitrarily small value (e.g. $-10^{10}$ ). The confidence bound $h$ is already available from the process monitoring model.

Then, iterate the following operations to traverse the upward solution tree in a standard depth-first search fashion, unless otherwise stated.

1. Examine $X_{m} \backslash X_{c}$. If the lower bound $\phi\left(X_{m} \backslash X_{c}\right)>h$, go to step 5; otherwise go to step 2 .

2. Examine $X_{m}$. If $\phi\left(X_{m}\right)<B$, prune this node (and its sub-nodes), and jump to the next iteration; otherwise go to step 3 .

3. $\forall x_{i} \in X_{c}$, examine $X_{m} \backslash x_{i}$. If $\phi\left(X_{m} \backslash x_{i}\right)>h$, jump to the next iteration at $X_{m} \backslash x_{i}$; otherwise go to step 4 .

4. $\forall x_{i} \in\left\{X_{m} \backslash X_{c}\right\}$, examine $X_{m} \backslash x_{i}$. If $\phi\left(X_{m} \backslash x_{i}\right)>h$, prune this node (and its sub-nodes), then jump to the next iteration; otherwise $X_{m}$ is a feasible and better solution to update $B=\phi\left(X_{m}\right)$, and then jump to the next iteration.

5. If $\phi\left(X_{m} \backslash X_{c}\right)<B$, prune node $X_{m}$ and all its brother nodes (and all the sub-nodes of them), then jump to the next iteration; otherwise move to step 6.

6. $\forall x_{i} \in\left\{X_{m} \backslash X_{c}\right\}$, examine $X_{m} \backslash\left\{X_{c} \cup x_{i}\right\}$. If $\phi\left(X_{m} \backslash\left\{X_{c} \cup x_{i}\right\}\right)>h$, prune node $X_{m}$ and all its brother nodes (and all the sub-nodes of them), jump to the next iteration; otherwise $X_{m} \backslash X_{c}$ is a feasible and better solution to update $B=\phi\left(X_{m} \backslash X_{c}\right)$, and then jump to the next iteration at next unvisited brother of its parent node.

\section{Application of RBMCA to PPCA mixture model for process monitoring}

PPCA is a probabilistic formulation of PCA proposed by Tipping and Bishop [23]. The main idea of PPCA is to project the original $n$-dimensional data $\mathbf{x}$ onto the principal subspace in which the variance is maximized: $\mathbf{x}=\mathbf{W t}+\boldsymbol{\mu}+\mathbf{e}$, where $\mathbf{W}$ is the loading matrix composing of the eigenvectors of the covariance matrix corresponding to the $q(q \leqslant n)$ largest eigenvalues, $\mathbf{t}$ is the score vector, $\boldsymbol{\mu}$ is the mean of sampled data and $\mathbf{e}$ is the noise term. 
Specifically, the noise $\mathbf{e}$ is assumed to be Gaussian with diagonal covariance matrix: $\mathbf{e} \sim \mathbf{G}\left(\mathbf{0}, \sigma^{2} \mathbf{I}\right)$, which implies $\mathbf{x} \mid \mathbf{t} \sim \mathbf{G}\left(\mathbf{W t}+\boldsymbol{\mu}, \sigma^{2} \mathbf{I}\right)$. Furthermore, by adopting a Gaussian prior distribution for the scores, $\mathbf{t} \sim \mathbf{G}(\mathbf{0}, \mathbf{I})$, the marginal distribution of the data is also Gaussian: $\mathbf{x} \sim \mathbf{G}(\boldsymbol{\mu}, \mathbf{C})$, where the covariance matrix is $\mathbf{C}=\mathbf{W} \mathbf{W}^{\mathrm{T}}+\sigma^{2} \mathbf{I}$.

PPCA can be extended to mixture models with $K$ components:

$$
\mathrm{p}(\mathbf{x})=\sum_{\mathrm{k}=1}^{\mathrm{K}} \lambda_{\mathrm{k}} \mathrm{G}\left(\boldsymbol{\mu}_{\mathrm{k}}, \mathbf{C}_{\mathrm{k}}\right)
$$

The sum of mixing weights must equal unity. Each mixture component is represented by a PPCA with mean $\boldsymbol{\mu}_{\mathrm{k}}$ and covariance matrix $\mathbf{C}_{\mathrm{k}}=\mathbf{W}_{\mathrm{k}} \mathbf{W}_{\mathrm{k}}^{\mathrm{T}}+\sigma_{\mathrm{k}}{ }^{2} \mathbf{I}$, where the $\mathbf{W}_{\mathrm{k}}$ is the loading matrix of order $n \times q_{k}$.

An iterative EM algorithm [24,25] can be implemented to estimate the model parameters $\boldsymbol{\mu}_{k}, \mathbf{C}_{k}, \lambda_{k}$ and $\sigma_{k}$. The issue of model selection refers to determining both the number of mixture components $(K)$ and the number of PCs within each mixture model $\left(q_{k}\right)$. Exhaustive search over all possible models is inefficient due to the large number of candidate models to be considered. A fast sub-optimal algorithm with Bayesian information criterion (BIC) $[14,26]$ was proposed to address the model selection problem.

In the community of MSPM, PPCA mixture model was introduced to monitor processes with multiple modes or non-Gaussian distributions [14,15]. The use of a fully probabilistic model provided a unified likelihood-based statistic $\mathrm{p}(\mathbf{x})$ for fault detection, as opposed to SPE and $\mathrm{T}^{2}$ in conventional methods. The sample data $\mathbf{x}$ is considered as out-of-control when the likelihood falls below the confidence bound: $\mathrm{p}(\mathbf{x})<h$, where the threshold $h$ determined by eq. (8) is the $100 \beta \%$ confidence:

$$
\int_{\mathbf{x}: \mathbf{p}(\mathbf{x})>h} \mathrm{p}(\mathbf{x}) \mathrm{d} \mathbf{x}=\beta
$$

This integral can be calculated using numerical methods such as Mote Carlo simulation [14].

In order to apply RBMCA to the PPCA mixture model, the following reconstruction problem needs to be solved:

$$
\mathrm{p}\left(\mathbf{x}_{m}^{*}, \mathbf{x}_{o}\right)_{\text {rec }}=\max _{\mathbf{x}_{m} \in \mathbf{R}^{d}} \mathrm{p}\left(\mathbf{x}_{m}, \mathbf{x}_{o}\right)
$$

Notice that reconstructing the missing variables is essentially a maximum likelihood estimation problem. It can be solved by using the expectation-maximization (EM) algorithm [25]. Specifically, define an auxiliary random variable $y$ to indicate from which mixture component the data is generated, and then the complete log-likelihood function is:

$$
L_{c}=\log \left(\mathrm{p}\left(\mathbf{x}_{m}, \mathbf{x}_{o}, y\right)\right)
$$

Given the current estimate of $\mathbf{x}_{m}$, denoted by $\mathbf{x}_{m}^{\text {old }}$, the expectation step (E-step) is to determine the expectation of $L_{c}$ with respect to $\mathrm{p}\left(y=k \mid \mathbf{x}_{m}^{\text {old }}, \mathbf{x}_{o}\right)$ :

$$
\begin{aligned}
& E\left(L_{c}\right)=\sum_{k=1}^{K}\left[\log \left(\mathrm{p}\left(\mathbf{x}_{m}, \mathbf{x}_{o}, y=k\right)\right) \times \mathrm{p}\left(y=k \mid \mathbf{x}_{m}^{\text {old }}, \mathbf{x}_{o}\right)\right] \\
& =\sum_{k=1}^{K}\left[\log \left(\mathrm{G}\left(\mathbf{x}_{m}, \mathbf{x}_{o} \mid \boldsymbol{\mu}_{k}, \mathbf{C}_{k}\right)\right) \times \mathrm{p}\left(y=k \mid \mathbf{x}_{m}^{\text {old }}, \mathbf{x}_{o}\right)\right]
\end{aligned}
$$

For brevity denote $\zeta_{k}=\mathrm{p}\left(y=k \mid \mathbf{x}_{m}^{\text {old }}, \mathbf{x}_{o}\right)$, which according to the Bayesian rule can be calculated as 


$$
\zeta_{k}=\mathrm{p}\left(y=k \mid \mathbf{x}_{m}^{\text {old }}, \mathbf{x}_{o}\right)=\frac{\mathrm{p}\left(\mathbf{x}_{m}^{\text {old }}, \mathbf{x}_{o} \mid y=k\right) \mathrm{p}(y=k)}{\sum_{k=1}^{K} \mathrm{p}\left(\mathbf{x}_{m}^{\text {old }}, \mathbf{x}_{o} \mid y=k\right) \mathrm{p}(y=k)}
$$

where $\mathrm{p}\left(\mathbf{x}_{m}^{\text {old }}, \mathbf{x}_{o} \mid y=k\right)$ is the probability of $\left(\mathbf{x}_{m}^{\text {old }}, \mathbf{x}\right)$ at the $k$-th mixture component and $\mathrm{p}(y=k)=\lambda_{k}$ is the prior probability of coming from the $k$-th mixture.

The maximization-step (M-step) is to find $\mathbf{x}_{m}$ in eq. (11) that maximizes $E\left(L_{c}\right)$. The necessary condition of the maximum is that the derivative of $E\left(L_{c}\right)$ with respect to $\mathbf{x}_{m}$ equals 0:

$$
\begin{aligned}
& \frac{\partial E\left(L_{c}\right)}{\partial \mathbf{x}_{m}}=\frac{\partial \sum_{k=1}^{K} \zeta_{k}\left[\log \left(\mathrm{G}\left(\mathbf{x}_{m}, \mathbf{x}_{o} \mid \boldsymbol{\mu}_{k}, \mathbf{C}_{k}\right)\right)\right]}{\partial \mathbf{x}} \times\left(\frac{\partial \mathbf{x}}{\partial \mathbf{x}_{m}}\right)^{T} \\
& =\frac{\partial\left[-\sum_{k=1}^{K} \zeta_{k} \frac{\left(\mathbf{x}-\boldsymbol{\mu}_{k}\right) \mathbf{C}_{k}^{-1}\left(\mathbf{x}-\boldsymbol{\mu}_{k}\right)^{T}}{2}\right]^{2} \times\left(\frac{\partial \mathbf{x}}{\partial \mathbf{x}_{m}}\right)^{T}}{=\left[-\sum_{k=1}^{K} \zeta_{k}\left(\mathbf{x}-\boldsymbol{\mu}_{k}\right) \mathbf{C}_{k}^{-1}\right] \times\left(\frac{\partial \mathbf{x}}{\partial \mathbf{x}_{m}}\right)^{T}=0}
\end{aligned}
$$

Furthermore, define the following block matrices:

$$
\begin{gathered}
\sum_{k=1}^{K} \zeta_{k} \boldsymbol{\mu}_{k} \mathbf{C}_{k}^{-1}=\mathbf{B}=\left[\mathbf{B}_{o}, \mathbf{B}_{m}\right] \\
\sum_{k=1}^{K} \zeta_{k} \mathbf{C}_{k}^{-1}=\mathbf{A}=\left(\begin{array}{ll}
\mathbf{A}_{o o} & \mathbf{A}_{o m} \\
\mathbf{A}_{m o} & \mathbf{A}_{m m}
\end{array}\right)
\end{gathered}
$$

Substitution of eqs. (14)(15) into eq. (13) gives

$$
\mathbf{x}_{o} \mathbf{A}_{o m}+\mathbf{x}_{m} \mathbf{A}_{m m}=\mathbf{B}_{m}
$$

Solving eq. (16) for $\mathbf{x}_{m}$ yields its estimate:

$$
\mathbf{x}_{m}^{n e w}=\left(\mathbf{B}_{m}-\mathbf{x}_{o} \mathbf{A}_{o m}\right) \mathbf{A}_{m m}^{-1}
$$

In summary, the EM algorithm to reconstruct the missing variables $\mathbf{x}_{m}$ runs as follows:

1. E-step: Given current estimate $\mathbf{x}_{m}^{\text {old }}$, update $\zeta_{k}=\mathrm{p}\left(y=k \mid \mathbf{x}_{m}^{\text {old }}, \mathbf{x}_{o}\right), k=\{1,2, \ldots K\}$, as in eq. (12).

2. M-step: Maximize $E\left(L_{c}\right)$ to obtain the new estimate $\mathbf{x}_{m}^{\text {new }}$; assign $\mathbf{x}_{m}^{\text {old }}=\mathbf{x}_{m}^{\text {new }}$ and return to the E-step until convergence.

To improve convergence, we do not use the original process data $\mathbf{x}$ for the initial estimate of $\mathbf{x}_{m}$ since it relates to a fault and may be far from the reconstructed value. Instead, we execute the M-step by using the prior probability $\lambda_{\mathrm{k}}$ in place of $\zeta_{k}$ to find a better initial estimate of the missing variables. With this method, the EM algorithm converges fairly quickly in the case studies reported in Section 4. The main computation involves the update of $\mathrm{p}\left(y \mid \mathbf{x}_{m}^{\text {old }}, \mathbf{x}_{o}\right)$ and the solution of a linear system (eq. (17)), both steps being computationally inexpensive.

The reconstruction procedure, through the EM algorithm, can be embedded into the generic RBMCV framework to search for the optimal combination of the faulty variables. 


\section{Case study}

In this section, fault isolation based on the proposed RBMCA method is demonstrated on an illustrative numeric example and the Tennessee Eastman benchmark problem. All computation is carried out using MATLAB ${ }^{\circledR}$ 2011a on a PC with Intel ${ }^{\circledR}$ Core $^{\mathrm{TM}}$ Duo CPU E8300 (2.83GHz).

\subsection{An illustrative example}

A numerical example with five variables $(\mathbf{x})$ and two internal states $(\mathbf{t})$ is studied to illustrate the fault isolation approach based on RBMVC analysis. The system is represented by:

$$
\mathbf{x}=\mathbf{N} \boldsymbol{\gamma}+\mathbf{v}
$$

where

$$
\mathbf{N}=\left(\begin{array}{ll}
-0.6589 & -1.4338 \\
0.8480 & -0.9921 \\
-0.7153 & 0.4654 \\
-1.5224 & 1.5562 \\
0.4028 & -0.2341
\end{array}\right), \quad \gamma=\left[\begin{array}{l}
\ln \left(\gamma_{1}^{2}\right) \\
\ln \left(\gamma_{2}^{2}\right)
\end{array}\right], \quad \gamma_{1} \sim \mathbf{G}(2,1), \quad \gamma_{2} \sim \mathbf{G}(5,1)
$$

Under NOC, the noise, $\mathbf{v}$, is normally distributed with zero mean and variance 0.1. Conventional PCA or single PPCA model is not expected to adequately represent the process variables under NOC due to the non-Gaussian distribution of the internal states. In this study, a PPCA mixture model is adopted.

First, 1000 data points under NOC are simulated by generating random samples for $\gamma$ and $\mathbf{v}$ from their corresponding distributions. A PPCA mixture model is then developed, whereby the BIC suggests the use of two mixtures, each having two principal components. The $99 \%$ confidence bound is $\log (h)=-4.52$. Notice that the monitoring statistic and its confidence bound are all shown in logarithm scale for ease of illustration. Accordingly, the $\log$-contribution is given as: $\log -\mathrm{RBMVC}_{\mathbf{x}_{m}}=\log \left(\mathrm{p}\left(\mathbf{x}_{m}^{*}, \mathbf{x}_{o}\right)_{\text {rec }}\right)-\log (\mathrm{p}(\mathbf{x}))$.

To simulate a fault, a step change is then introduced to the 2 nd and 4 th variables with the same magnitude. Two step values, 2 and 4, are considered to investigate the impact of the magnitude of the fault. In both cases, the fault is immediately detected; the $\log$ monitoring statistics $(\log (\mathrm{p}(\mathbf{x})))$ are -10.41 (magnitude $=2$ ) and -30.29 (magnitude=4), both less than the $99 \%$ confidence bound. For the purpose of fault isolation, the log-contribution of individual variables, as defined above, is shown in Figs. 2 and 3, where the control limit is plotted in terms of $(\log (h)-\operatorname{logp}(\mathbf{x}))$. For both cases, no single variable's contribution exceeds the confidence bound, suggesting that univariate contribution does not unambiguously reveal the faulty variables. It may be argued that Fig. 3, corresponding to fault magnitude 4, well identifies the 2 nd and 4 th variables as faulty. Nonetheless, when the fault magnitude reduces to 2 , as illustrated in Fig. 2, one cannot easily rule out the possibility that the 1 st variable is also responsible to the fault.

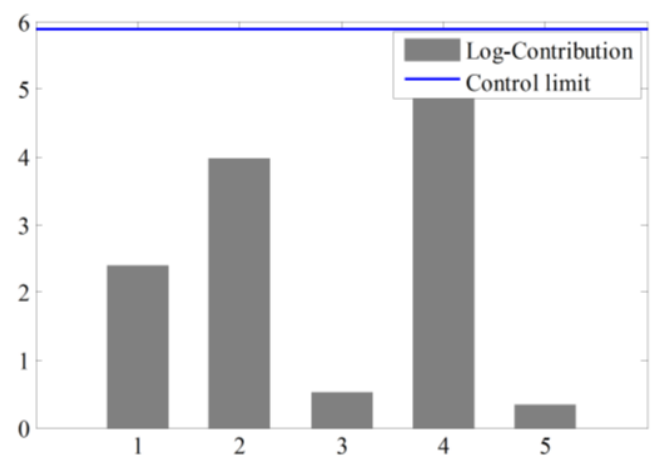

Fig. 2. The contribution of individual variables to a step fault (magnitude=2). 


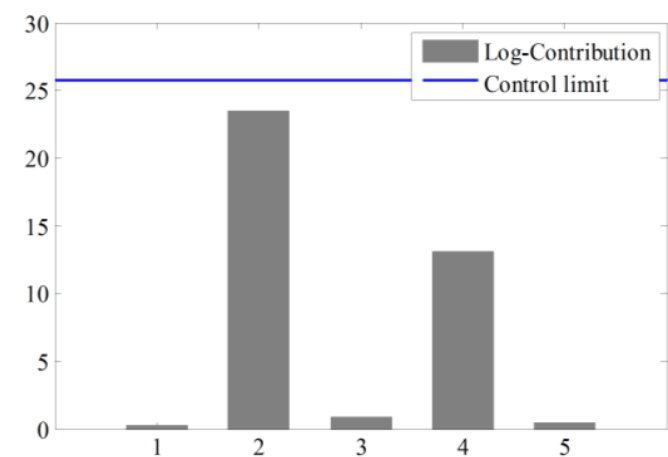

Fig. 3. The contribution of individual variables to a step fault (magnitude=4).

While univariate contribution analysis does not clearly identify faulty variables, the proposed RBMVC method does. The $\mathrm{BAB}$ algorithm correctly attributes the fault to the combination of the 2 nd and 4 th variables in both cases. For comparison, Table 1 lists the reconstructed log-likelihood (monitoring statistic) and the log-RBMVC of all combinations of two variables. Note that not all these combinations are actually examined in the BAB algorithm. For a small fault magnitude of 2 , reconstructing five combinations of two variables may bring the process back to normal (in bold in the table); yet $\left\{x_{2}, x_{4}\right\}$ is still isolated due to the largest log-RBMVC. For fault magnitude of 4 , this combination is the only feasible one to be identified as faulty (reconstructed log-likelihood greater than the monitoring confidence bound).

Table 1. Reconstructed log-likelihood and log-RBMVC for all two-variable combinations. The $99 \%$ confidence bound for $\log$-likelihood is -4.52 , and that for $\log$-RBMVC is 5.89 (fault magnitude $=2$ ) and 25.77 (fault magnitude $=4$ ).

\begin{tabular}{|c|c|c|c|c|c|}
\hline \multicolumn{3}{|c|}{ Fault magnitude $=2$} & \multicolumn{3}{|c|}{ Fault magnitude $=4$} \\
\hline $\begin{array}{l}\text { Missing } \\
\text { variable }\end{array}$ & $\begin{array}{l}\text { Reconstructed } \\
\text { log-likelihood }\end{array}$ & $\log -\mathrm{RBMVC}$ & $\begin{array}{l}\text { Missing } \\
\text { variable }\end{array}$ & $\begin{array}{l}\text { Reconstructed } \\
\text { log-likelihood }\end{array}$ & $\log -\mathrm{RBMVC}$ \\
\hline$\left\{x_{1}, x_{2}\right\}$ & -2.90 & 7.51 & $\left\{x_{1}, x_{2}\right\}$ & -5.72 & 24.57 \\
\hline$\left\{x_{1}, x_{3}\right\}$ & -7.99 & 2.42 & $\left\{x_{1}, x_{3}\right\}$ & -29.44 & 0.85 \\
\hline$\left\{x_{1}, x_{4}\right\}$ & -3.89 & 6.52 & $\left\{x_{1}, x_{4}\right\}$ & -16.65 & 13.64 \\
\hline$\left\{x_{1}, x_{5}\right\}$ & -8.01 & 2.40 & $\left\{x_{1}, x_{5}\right\}$ & -29.88 & 0.41 \\
\hline$\left\{x_{2}, x_{3}\right\}$ & -6.44 & 3.97 & $\left\{x_{2}, x_{3}\right\}$ & -6.47 & 23.82 \\
\hline$\left\{x_{2}, x_{4}\right\}$ & -0.11 & 10.29 & $\left\{x_{2}, x_{4}\right\}$ & 1.12 & 31.41 \\
\hline$\left\{x_{2}, x_{5}\right\}$ & -6.36 & 4.05 & $\left\{x_{2}, x_{5}\right\}$ & -6.02 & 24.27 \\
\hline$\left\{x_{3}, x_{4}\right\}$ & -3.54 & 6.87 & $\left\{x_{3}, x_{4}\right\}$ & -13.89 & 16.40 \\
\hline$\left\{x_{3}, x_{5}\right\}$ & -9.77 & 0.63 & $\left\{x_{3}, x_{5}\right\}$ & -29.15 & 1.14 \\
\hline$\left\{x_{4}, x_{5}\right\}$ & -4.18 & 6.23 & $\left\{x_{4}, x_{5}\right\}$ & -15.46 & 14.83 \\
\hline
\end{tabular}

Furthermore, the impact of the total number of process variables $(n)$ on computation is investigated. Five cases are considered $(n=10,20, \cdots, 50)$. The linear system to simulate the process still follows eqs. (18) and (19) with two internal states, except that the $n \times 2$ matrix $\mathbf{N}$ is randomly generated. Then, a step change (magnitude $=8$ ) is introduced in five randomly selected process variables to simulate a fault. For each case, the fault isolation procedure is repeated 100 times and the results are summarized in Table 2, including the number of visited nodes, the computation time, and fault isolation accuracy. Accuracy is the percentage that the faulty variables are isolated correctly. In comparison with the original combinatorial problem, the number of nodes evaluated is dramatically reduced, resulting in saving of computation time. Even in the presence of 50 variables, the average computation time is still less than one minute, suggesting the on-line fault isolation capability of the proposed method. In addition, the RBMCA has correctly 
identified the faulty variables in most cases.

Table 2. The impact of total number of process variables on computation and accuracy.

\begin{tabular}{ccccccccc}
\hline Case & $\begin{array}{c}\text { No. } \\
\text { candidate } \\
\text { nodes }\end{array}$ & $\begin{array}{c}\text { No. visited } \\
\text { nodes } \\
\text { (average) }\end{array}$ & $\begin{array}{c}\text { No. visited } \\
\text { nodes } \\
(\max )\end{array}$ & $\begin{array}{c}\text { No. visited } \\
\text { nodes } \\
(\min )\end{array}$ & $\begin{array}{c}\text { Time } \\
\text { (average) }\end{array}$ & $\begin{array}{c}\text { Time } \\
(\max )\end{array}$ & $\begin{array}{c}\text { Time } \\
(\min )\end{array}$ & Accuracy \\
\hline$n=10$ & 1024 & 7.09 & 14 & 6 & $0.06 \mathrm{~s}$ & $0.14 \mathrm{~s}$ & $0.02 \mathrm{~s}$ & $100 \%$ \\
$n=20$ & 1048576 & 40.05 & 329 & 6 & $0.55 \mathrm{~s}$ & $6.33 \mathrm{~s}$ & $0.06 \mathrm{~s}$ & $100 \%$ \\
$n=30$ & $1.08 \times 10^{9}$ & 46.04 & 191 & 6 & $1.03 \mathrm{~s}$ & $3.18 \mathrm{~s}$ & $0.16 \mathrm{~s}$ & $95 \%$ \\
$n=40$ & $1.10 \times 10^{12}$ & 136.5 & 505 & 11 & $7.63 \mathrm{~s}$ & $18.11 \mathrm{~s}$ & $0.91 \mathrm{~s}$ & $92 \%$ \\
$n=50$ & $1.13 \times 10^{15}$ & 187.13 & 987 & 6 & $19.87 \mathrm{~s}$ & $132.06 \mathrm{~s}$ & $0.39 \mathrm{~s}$ & $90 \%$ \\
\hline
\end{tabular}

The computation is also affected by the number of variables that are actually responsible for the detected fault. For $n=50$, we vary the number of variables that deviate from NOC due to step change (magnitude=8), and the results are given in Fig. 4 (averaged over 100 random simulations of each case). Even for the worst case of 15 faulty variables, the average computation time is less than three minutes, and the average number of visited nodes is only a fraction of the total number of candidates $\left(2^{50} \approx 1.13 \times 10^{15}\right)$.

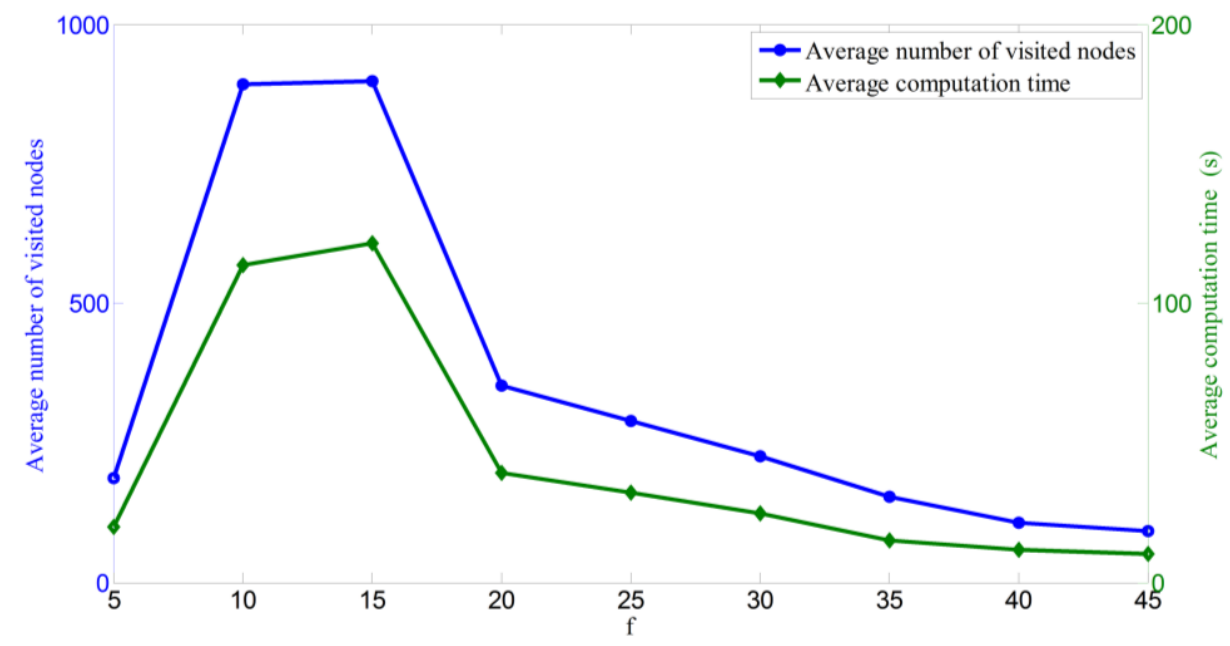

Fig. 4. The impact of number of faulty variables on computation $(n=50)$.

\subsection{The Tennessee Eastman process}

The Tennessee Eastman (TE) process is a benchmark problem for testing process control and MSPM methods [27]. The flow sheet of the TE process is reproduced in Fig. 5. This process produces two products $(G$ and $H)$ from four reactants $(A, C, D$ and $E$ ). A byproduct $F$ is also produced. In addition, an inert component $B$ also presents in $C$ stream (stream 4) and in trace amount in $A$ feed stream (stream 1). The plant has five units: reactor, condenser, separator, stripper and compressor. The decentralized control strategy developed by Ricker [28] is used in this study to regulate the steady-state operation. There are a total of 41 measurements and 12 manipulated variables available. For simplicity, only 22 continuous measurements, listed in Table 3, are selected for process monitoring and fault isolation. Five typical faulty modes, listed in Table 4, are simulated to illustrate the fault isolation approach. The isolation results can be validated by analyzing the underlying process operation according to the described root cause of these faults in the original paper [27]. 


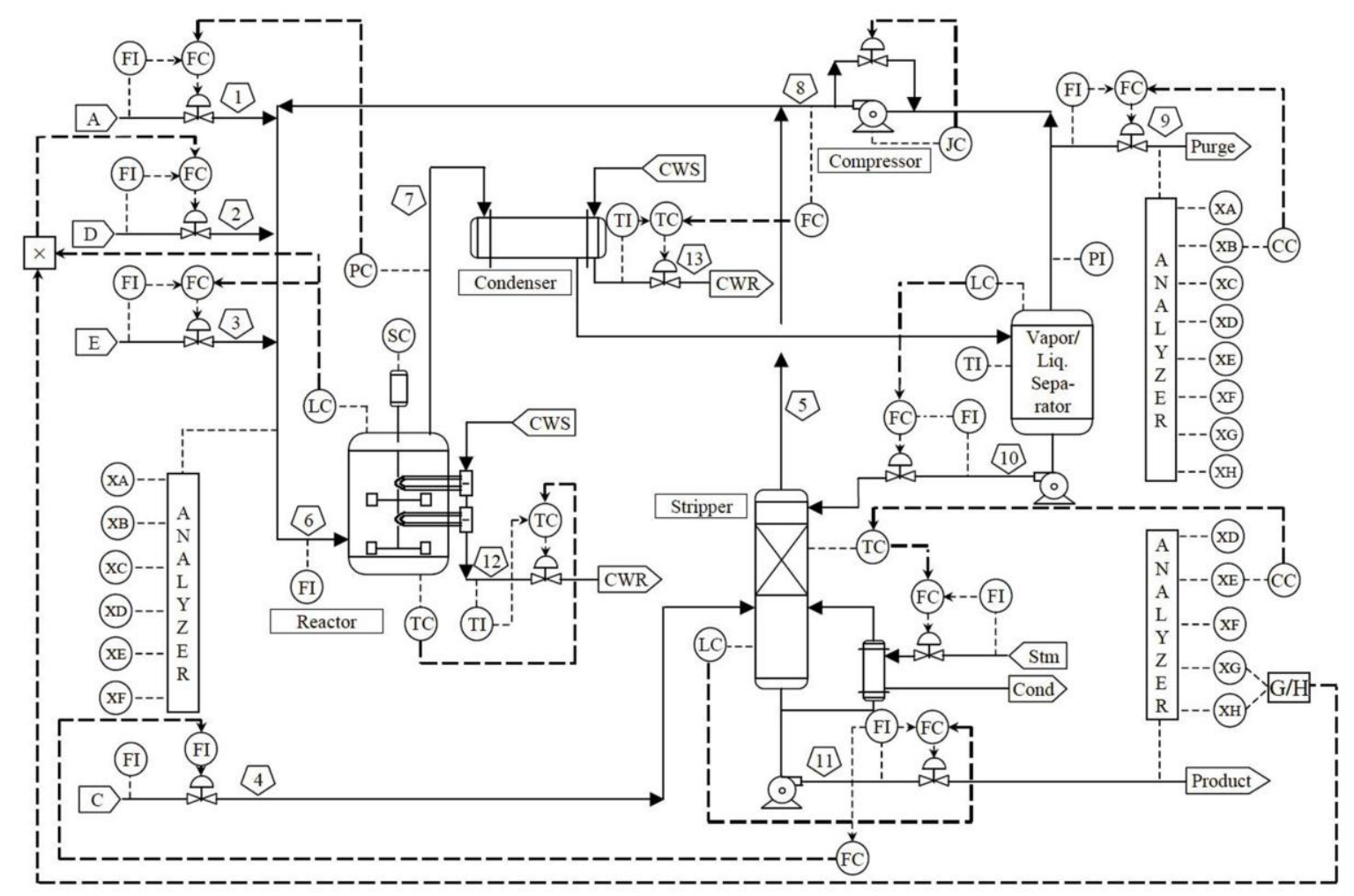

Fig. 5. Flow sheet of the TE process.

Table 3. Continuously measured variables.

\begin{tabular}{lclc}
\hline ID & Description & ID & Description \\
\hline$x_{1}$ & A Feed(Stream1) & $x_{12}$ & Product Separator Level \\
$x_{2}$ & D Feed(Stream2) & $x_{13}$ & Product Separator Pressure \\
$x_{3}$ & E Feed(Stream3) & $x_{14}$ & Product Separator Underflow(Stream 10) \\
$x_{4}$ & A and C Feed(Stream4) & $x_{15}$ & Stripper Level \\
$x_{5}$ & Recycle Flow(Stream 8) & $x_{16}$ & Stripper Pressure \\
$x_{6}$ & Reactor Feed Rate(Stream6) & $x_{17}$ & Stripper Underflow(Stream 11) \\
$x_{7}$ & Reactor Pressure & $x_{18}$ & Stripper Temperature \\
$x_{8}$ & Reactor Level & $x_{19}$ & Stripper Stream Flow \\
$x_{9}$ & Reactor Temperature & $x_{20}$ & Compressor Work \\
$x_{10}$ & Purge Rate(Stream9) & $x_{21}$ & Reactor Cooling Water Outlet Temperature \\
$x_{11}$ & Product Separator Temperature & $x_{22}$ & Condenser Cooling Water Outlet Temperature \\
\hline
\end{tabular}

Table 4. Operational faults.

\begin{tabular}{lcc}
\hline Fault ID & Description & Type \\
\hline 2 & Step in B composition whilst A/C ratio is constant(stream 4) & Step \\
4 & Step in Reactor Cooling Water Inlet Temperature & Step \\
6 & A Feed Loss (stream 1) & Step \\
7 & C Header Pressure Loss-reduced availability (stream 4) & Step \\
$12+15$ & Random variation in Condenser Cooling Water inlet Temperature & Variation \\
& and Sticking Condenser Cooling Water Valve & + Sticking \\
\hline
\end{tabular}

Two thousand samples under NOC were simulated with sampling interval of $0.01 \mathrm{hr}$. Then, additional 2000 samples under NOC were produced to assess the false alarm rate. Further, the process was run under each fault mode for 4 hours, giving rise to 400 faulty samples per mode. Fig. 6 gives the quantile-quantile (Q-Q) plots for the PCA 
scores corresponding to the four largest eigenvalues versus the standard Gaussian distribution. It appears that Gaussian distribution may be inadequate to describe the PCA scores, especially those corresponding to the largest three largest eigenvalues. Consequently, a PPCA mixture model is developed to ensure effective process monitoring and fault isolation. As discussed in Section 3, the model parameters are estimated using the EM algorithm, with two mixtures (10 and 11 principal components) as determined by the BIC.

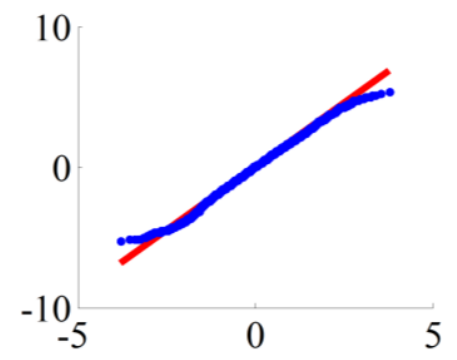

(a)

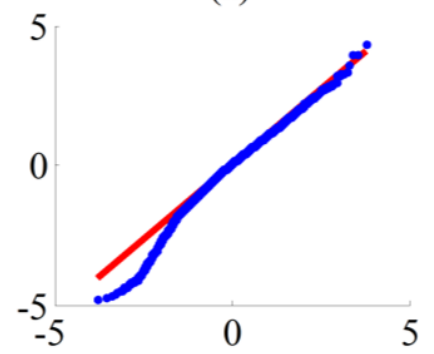

(c)

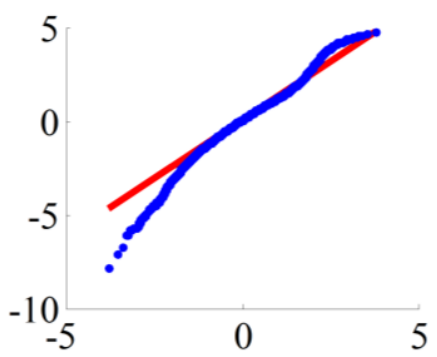

(b)

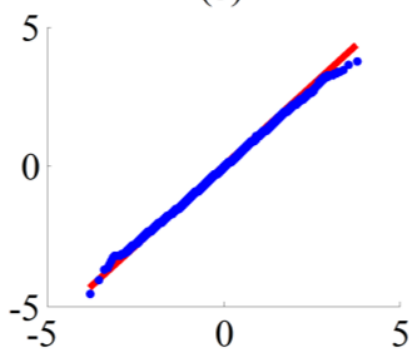

(d)

Fig. 6. Q-Q plots. The horizontal axes are the quantiles of a standard Gaussian distribution and the vertical axes are the quantiles of the PCA scores corresponding to the largest four eigenvalues: (a) 1st, (b) 2nd, (c) 3rd, (d) 4th.

First, Table 5 compares fault detection capability of traditional PCA (using Hotelling's $\mathrm{T}^{2}$ and SPE) with the more sophisticated PPCA mixture model. For both methods, the 99\% confidence bound was used. The false alarm rate of conventional PCA (7.6\%) is much higher than the theoretical value (1\%), due to non-Gaussian data distribution. In contrast, the false alarm rate of PPCA mixture model is more reasonable. Nevertheless, PPCA mixture model outperformed conventional PCA in terms of detection delay only for Fault 2; they gave the same results for the other faults. This may be explained by the fact that the four faults (other than Fault 2) have significant and immediate impact on process variables and can be quickly detected by both methods, while Fault 2 is more subtle and detected earlier by the PPCA mixture model.

Table 5. Fault detection results.

\begin{tabular}{ccccccc}
\hline Model & False alarm & \multicolumn{4}{c}{ Detection delay (hr) } \\
& rate (\%) & Fault 2 & Fault 4 & Fault 6 & Fault 7 & Fault 12+15 \\
\hline PCA model & 7.6 & 0.83 & 0.00 & 0.00 & 0.00 & 0.20 \\
PPCA mixture model & 1.5 & 0.68 & 0.00 & 0.00 & 0.00 & 0.20 \\
\hline
\end{tabular}

The RBMCA results for fault isolation are shown in Table 6. In all cases, the BAB algorithm takes no more than a few seconds to pinpoint the most responsible variables, indicating its suitability for time-critical on-line applications. The $\mathrm{BAB}$ is extremely efficient, since only a small fraction of the total $2^{22} \approx 4.2 \times 10^{6}$ nodes has been examined to solve the combinatorial optimization problem. In addition, no more than two variables have been selected to explain the faults, which is helpful for further analysis. 
Table 6. Fault isolation results of TE process by using the RBMVC method.

\begin{tabular}{cccc}
\hline Fault ID & $\begin{array}{c}\text { Identified } \\
\text { variables }\end{array}$ & Number of visited nodes & Computation time \\
\hline Fault 2 & $\left\{x_{10}\right\}$ & 140 & $2.53 \mathrm{~s}$ \\
Fault 4 & $\left\{x_{9}, x_{21}\right\}$ & 3 & $0.08 \mathrm{~s}$ \\
Fault 6 & $\left\{x_{1}\right\}$ & 54 & $0.52 \mathrm{~s}$ \\
Fault 7 & $\left\{x_{4}\right\}$ & 2 & $0.06 \mathrm{~s}$ \\
Fault 12+15 & $\left\{x_{22}\right\}$ & 2 & $0.08 \mathrm{~s}$ \\
\hline
\end{tabular}

Moreover, reconstruction based univariate contribution analysis is carried out for Fault 4(all other faults have a single isolated variable). The reconstructed $22 \mathrm{log}$-likelihood values, corresponding to 22 process variables, are all far below the $99 \%$ confidence bound of -41.31 ; numerically they are all $-\infty$ except the value for $x_{21}(-169.51)$. In contrast, by isolating $x_{9}$ and $x_{21}$, the reconstructed log-likelihood is -29.24 , clearly indicating the dominant influence of these two variables combined.

As comparison, conventional PCA-based univariate contribution analysis is carried out for Fault 4. It was detected on both SPE and $\mathrm{T}^{2}$ charts, and Fig. 7 shows the corresponding contribution plots. It may be inferred from SPE contribution plot that $x_{10}$ and $x_{21}$ have the biggest contribution, while the $\mathrm{T}^{2}$ contribution plot points to $x_{9}$ and $x_{10}$. Moreover, it is very difficult to determine whether these three variables, when combined, are the dominant source of fault. Even the contribution of $x_{4}$ is not insignificant. This ambiguity, with regard to which combination of variables should be isolated so that the process would return to normal, is exactly the issue that the proposed RBMCA addresses. RBMCA is capable of isolating as few as possible, but essential, faulty variables to facilitate further fault diagnosis.
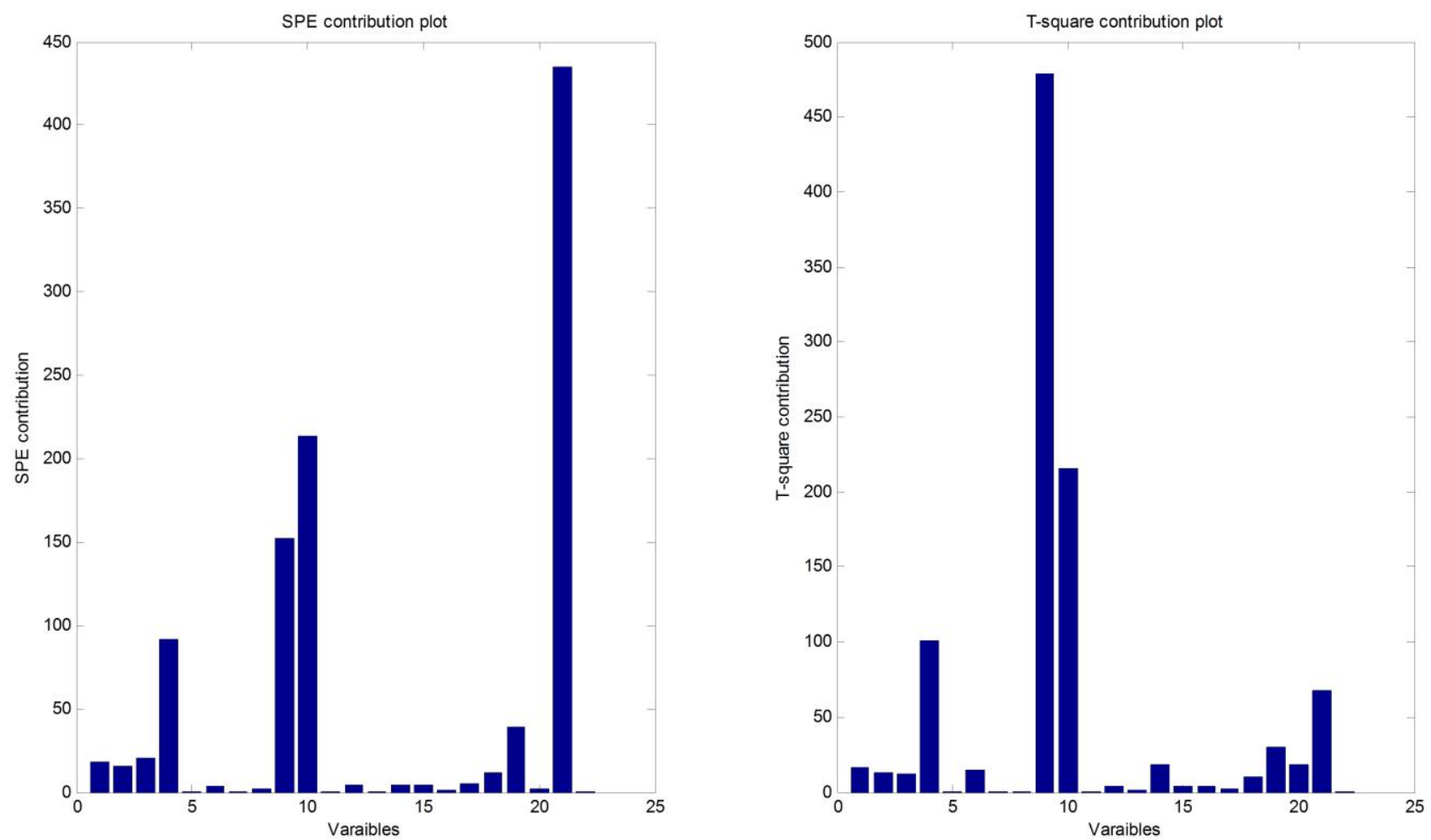

Fig. 7. SPE and $\mathrm{T}^{2}$ contribution plots of fault 4 .

A close inspection of process operation confirms that the RBMVC results are reasonable. For fault 2, a step change in B composition (stream 4) increases the amount of this inert component in the plant. B is non-condensable and must exit from the purge stream 9, increasing the purge rate $x_{10}$. For fault 4 , step change in reactor cooling water 
inlet temperature directly leads to abnormal variation of reactor temperature $x_{9}$ and reactor cooling water outlet temperature $x_{21}$. It is clear that fault 6 (A feed loss) has significant influence on the flow rate of component A in stream $1\left(x_{1}\right)$. Fault 7 is to do with $\mathrm{C}$ header pressure loss (reduced availability) in stream 4, resulting in decrease in the total feed from stream $4\left(x_{4}\right)$. Finally, fault $12+15$ is due to random variation in condenser cooling water inlet temperature and sticking condenser cooling water valve, giving rise to abnormal temperature variation in the outlet cooling water of the condenser $\left(x_{22}\right)$.

Furthermore, while the process is approximately static at steady-state, it is not so when a fault occurs. The process usually deviates from its current steady-state until it reaches a new one. This process dynamics is likely to cause the fault to propagate from some variables to others. Fault propagation can also be analyzed by using the RBMVC method to examine how the set of faulty variables change over time. Table 7 shows two typical examples.

Table 7. Propagation of faulty variables.

\begin{tabular}{|c|c|c|c|}
\hline $\begin{array}{c}\text { Fault } \\
\text { ID }\end{array}$ & Identified variables & $\begin{array}{l}\text { Average no. of } \\
\text { visited nodes }\end{array}$ & Average time \\
\hline Fault 6 & $\left\{x_{1}\right\} \rightarrow\left\{x_{1}, x_{7}, x_{16}, x_{21}\right\} \rightarrow\left\{x_{1}, x_{7}, x_{10}, x_{11}, x_{13}, x_{16}, x_{21}, x_{22}\right\}$ & 2621.52 & $66.48 \mathrm{~s}$ \\
\hline Fault 7 & $\begin{aligned}\left\{x_{4}\right\} \rightarrow\left\{x_{4}, x_{7}, x_{9}, x_{11}, x_{13}, x_{16}, x_{18}, x_{21}\right\} \rightarrow\left\{x_{7}, x_{10}, x_{11}, x_{13}, x_{16}, x_{18}, x_{22}\right\} & \rightarrow\left\{x_{7}, x_{10}, x_{13}, x_{16}\right\} \rightarrow\left\{x_{10}\right\}\end{aligned}$ & 946.71 & $28.58 \mathrm{~s}$ \\
\hline
\end{tabular}

The propagation of faulty variables may be physically explained as follows. Fault 6 is a challenge to the plant since it involves the loss of A feed, a major anomaly. The flow rate of A component in stream $1\left(x_{1}\right)$ is immediately affected. Then, the imbalance of reactants causes a rapid pressure increase in the reactor $\left(x_{7}\right)$ and later in the stripper $\left(x_{16}\right)$. Meanwhile, subdued reaction due to lack of reactant A releases less heat (main reactions are exothermic), and thus the control action maintaining reactor temperature leads to change of cooling water outlet temperature $\left(x_{21}\right)$. A short time later, the fault further progresses to several other variables, and the process settles at a new steady-state [28].

Fault 7 involves $\mathrm{C}$ header pressure loss (reduced availability) in stream 4, resulting in a decrease in the total feed in stream $4\left(x_{4}\right)$. Then, this disturbance causes variation in several pressure and temperature variables due to insufficient reaction, such as $x_{7}, x_{9}, x_{11}, x_{13}, x_{16}$ and $x_{18}$, and later in cooling water temperatures $\left(x_{21}, x_{22}\right)$. A short time later, $x_{4}$ returns to normal level due to the action of flow control. Meanwhile, the reactor temperature control loop brings $x_{9}$ back to normal. Similarly, other temperature and pressure variables go back to normal later and only the purge rate $\left(x_{10}\right)$ is left. The purge rate is higher than normal to remove excessive by-products that are the result of insufficient main reactions. Such a sequence of events, as manifested by the propagation of faulty variables, would be very helpful to pinpoint the root-cause of the disturbance, i.e. $\mathrm{C}$ header pressure loss.

\section{Conclusions}

In this paper, a generic framework of reconstruction based multivariate contribution analysis (RBMCA) has been proposed for isolating faulty variables. Reconstructed values of missing variables are chosen to optimize the monitoring statistic. The variables are considered to be faulty if the reconstruction would bring the monitoring statistic, and thus the process, back to normal condition. In order to determine the most responsible variables, the RBMCA is formulated as a combinatorial optimization problem and is efficiently solved by using the BAB algorithm. We demonstrate the application of the RBMCA to a specific process monitoring method, the PPCA mixture model; nevertheless the generic formulation allows the proposed framework to be applicable to any statistical models. The computational efficiency and fault isolation capability are confirmed through a numerical example, as well as the simulated TE process. It is interesting to observe how the fault propagates due to process dynamics as identified by 
RBMCA. The time-sequence of isolated variables will be very helpful to diagnose the root cause of the detected fault. Currently, we are developing a formal way to exploit the fault propagation information for diagnosis and root cause analysis purposes.

\section{References}

[1] Y. Hu, D.H. Zhou, Fault diagnosis techniques for dynamic systems, Acta Automatica Sinica 35(6) (2009) 748-758.

[2] V. Venkatasubramanian, R. Rengaswamy, K. Yin, S.N. Kavuri, A review of process fault detection and diagnosis part I: Quantitative model-based methods, Computers and Chemical Engineering 27(3) (2003) 293-311.

[3] V. Venkatasubramanian, R. Rengaswamy, K. Yin, S.N. Kavuri, A review of process fault detection and diagnosis part III: Process history based method, Computers and Chemical Engineering 27(3) (2003) 327-346.

[4] E.B. Martin, A.J. Morris, J. Zhang, Process performance monitoring using multivariate statistical process control, IEE Proceedings: Control Theory and Applications, 143(2) (1996) 132-144.

[5] J.F. MacGregor, T. Kourti, Statistical process control of multivariate processes. Control Engineering Practice 3 (1995) 403-414.

[6] A. AlGhazzawi, B. Lennox, Monitoring a complex refining process using multivariate statistics 16 (2008) 294-307

[7] S.J. Qin, Statistical process monitoring: basics and beyond, Journal of Chemometrics, 17(8-9) (2003) 480-502.

[8] E.B. Martin, A.J. Morris, Non-parametric confidence bounds for process performance monitoring charts, Journal of process control 6 (1996) 349-358.

[9] U. Thissen, H. Swierenga, A.P. de Weijer, R. Wehrens, W.J. Melssen, L.M.C. Buydens, Multivariate statistical process control using mixture modeling, Journal of Chemometrics 19 (2005) 23-31.

[10] J. Chen, J. Liu, Using mixture principal component analysis networks to extract fuzzy rules from data, Industrial and Engineering Chemistry Research 29 (2000) 2355-2367.

[11] T. Chen, J. Zhang, On-line multivariate statistical monitoring of batch processes using Gaussian mixture model. Computers and Chemical Engineering 34 (2010) 500-507.

[12] M. Kano, S. Tanaka, S. Hasebe, I. Hashimoto, H. Ohno, Monitoring independent components for fault detection. AIChE Journal 49 (2003) 969-976

[13] Z. Ge, Z. Song, A distribution-free method for process monitoring. Expert Systems with Applications 38 (2011) 9821-9829.

[14] T. Chen, Y. Sun, Probabilistic contribution analysis for statistical process monitoring: A missing variable approach. Control Engineering Practice 17 (2009) 469-477.

[15] S.W. Choi, E.B. Martin, A.J. Morris, Fault detection based on a maximum-likelihood principal component analysis (PCA) mixture, Industrial and Engineering Chemistry Research 44 (2005) 2316-2327.

[16] D. Leung, J. Romagnoli, An integration mechanism for multivariate knowledge-based fault diagnosis. Journal of Process Control 12(1) (2002) 15-26.

[17] P. Miller, R.E. Swanson, C.F. Heckler. Contribution plots: A missing link in multivariate quality control. International Journal of Applied Mathematics and Computer Science 8 (1998) 775-792.

[18] J.A. Weterhuis, S.P. Gurden, A.K. Smilde. Generalized contribution plots in multivariate statistical process monitoring. Chemometrics and Intelligent Laboratory Syatem 51 (2000) 95-114.

[19] R. Dunia, S.J. Qin, T. Edgar, T. McAvoy. Identification of faulty sensors using PCA. AIChE Journal 42 (1996) 2797-2812.

[20] C.F. Alcala, S.J. Qin. Reconstruction-based contribution for process monitoring. Automatica 45 (2009) 1593-1600.

[21] G. Li, C.F. Alcala, S.J. Qin, D.H. Zhou, Generalized reconstruction-based contributions for output-relevant fault 
diagnosis with application to the Tennessee Eastman process, IEEE transactions on control systems technology 19(5) (2011) 1114-1127.

[22] V. Kariwala, P.E. Odiowei, Y. Cao, T. Chen, A branch and bound method for isolation of faulty variables through missing variable analysis, Journal of Process Control 20 (2010) 1198-1206.

[23] M.E. Tipping, C.M. Bishop, Probabilistic principal component analysis, Journal of the Royal Statistical Society B 61 (1999) 611-622.

[24] M.E. Tipping, C.M. Bishop, Mixtures of probabilistic principal component analysers, Neural Computation 11(2) (1999) 443-482.

[25] A.P. Dempster, N.M. Laird, D.B. Rubin, Maximum likelihood from incomplete data via the EM algorithm (with discussion), Journal of the Royal Statistical Society. Series B (Methodological) 39(1) (1977) 1-38.

[26] M.E.M. Musa, D. de Ridder, R.P.W. Duin, V. Atalay, Almost autonomous training of mixtures of principal component analyzers. Pattern Recognition Letters 25 (2004) 1085-1095.

[27] J.J. Downs, E.F. Vogel. A plant-wide industrial process control problem, Computers and Chemical Engineering 17(3) (1993) 245-255.

[28] N.L. Ricker. Decentralized control of the Tennessee Eastman Challenge Process, Journal of Process Control 6(4) (1996) 205-221. 